\title{
Tradução e adaptação transcultural do instrumento de avaliação do profissionalismo P-MEX para uso em médicos residentes
}

Translation and transcultural adaptation of the Professional Mini-Evaluation Exercise (P-MEX) for use on medical residents

\author{
Mariana Matias de Lima Holdefer ${ }^{1}$ (D) marianaholdefer@gmail.com \\ Cláudia Fonseca Sena' (1D) claudiafonseca7@hotmail.com \\ Alessandra Vitorino Naghettini' ${ }^{1}$ (D) anaghettini@gmail.com \\ Edna Regina Silva Pereira' (1) |ersp13@gmail.com
}

\begin{abstract}
RESUMO
Introdução: Profissionalismo médico refere-se a vários atributos, valores, comportamentos, responsabilidades e compromissos dos médicos com os pacientes e com a sociedade. O profissionalismo é traduzido, hoje, como uma nova competência agregada ao conjunto de habilidades médicas, devendo ser demonstrada, ensinada e avaliada durante a formação desses profissionais. O Professionalism Mini-Evaluation Exercise (P-MEX) é um instrumento de avaliação do profissionalismo médico criado no Canadá, em 2006, e já validado para uso em alguns países, mostrando evidências de validade, confiabilidade e reprodutibilidade dos resultados nesses lugares.
\end{abstract}

Objetivo: O objetivo deste estudo foi desenvolver uma versão do instrumento em língua portuguesa e adaptada transculturalmente para uso no Brasil.

Método: A tradução e a adaptação transcultural foram realizadas seguindo as diretrizes da International Test Commission (ITC) - segunda versão 2017. Adotaram-se as seguintes etapas: tradução para o português por dois médicos brasileiros fluentes na língua inglesa, revisão da tradução por um comitê revisor, retrotradução por dois professores de inglês procedentes de países de língua inglesa, revisão da retrotradução por um comitê revisor, aprovação da retrotradução pelo autor original do questionário e, por último, aplicação da versão consenso final do instrumento ao público-alvo da pesquisa para avaliar a clareza, compreensão e aceitabilidade.

Resultados: A versão final em português foi considerada adequada e utilizada como definitiva, constituindo a versão final em português do P-MEX. Todo o processo realizado seguindo guias internacionais classicamente utilizados, inclusive a aprovação final do autor do instrumento original, reitera que a nova versão adaptada do questionário tem validade de conteúdo correspondente ao original.

Conclusão: Diante da lacuna de instrumentos para medir o profissionalismo médico no Brasil, realizaram-se a tradução e a adaptação transcultural do P-MEX para uso no país, podendo ser utilizado para estimular uma prática profissional mais adequada para os pacientes e a sociedade.

Palavras-chave: Educação Médica; Profissionalismo; Tradução; Estudos de Validação.

\begin{abstract}
Introduction: Medical Professionalism refers to several attributes, values, behaviors, responsibilities and commitments of physicians in relation to their patients and society. Professionalism translates today as a new competence composed of the medical skill set that should be demonstrated, taught and evaluated during the training of these professionals. The Professionalism Mini-Evaluation Exercise (P-MEX) is a medical professionalism evaluation tool created in Canada in 2006 and validated for use in Japan, Finland and Iran, having demonstrated its validity, reliability and reproducibility in these countries.

Objective: The objective of this study is to develop a version of this instrument in Portuguese and adapt it transculturally to be used in Brazil.

Method: The translation and transcultural adaptation was made according to the International Test Commission (ITC) - $2^{\text {nd }}$ edition 2017 guidelines. The following steps were taken: translation into Portuguese by two fluent English-speaking Brazilian physicians, review of the translation by a Review Committee, backtranslation by two English teachers from English speaking countries, review of the backtranslation by a Review Committee, approval of the backtranslation by the original author of the questionnaire and, finally, application of the agreeded final form of the instrument to the target population to evaluate its clarity, understandability and acceptability.
\end{abstract}

Results: The final Portuguese form was considered suitable, constituting the final Portuguese version of the Professionalism Mini-Evaluation Exercise $(P-M E X)$. The entire process was conducted in accordance with classically used international guidelines, including the final approval of the author of the original form, reasserting that the validity of the new adapted version of the form matches that of the original.

Conclusion: In view of the lack of instruments to measure medical professionalism in Brazil, translation and cross-cultural adaptation of the P-MEX for use in the country was carried out, to be used to stimulate a more appropriate professional practice for patients and society.

Keywords: Medical Education; Professionalism; Translating; Validation Studies.

${ }^{1}$ Universidade Federal de Goiás, Goiânia, Goiás, Brasil.

Editora-chefe: Daniela Chiesa

Editor associado: Rosiane Viana Zuza Diniz

Recebido em 05/09/20; Aceito em 05/01/21.

Avaliado pelo processo de double blind review. 


\section{INTRODUÇÃO}

A cada dia são processados 70 médicos no Brasil, três novas ações judiciais por hora, de acordo com os dados do Conselho Nacional de Justiça (CNJ)1. São mais de 26 mil processos anuais que representam $7 \%$ de toda a categoria. O crescimento expressivo do número de demandas judiciais por erro médico e pela má prática no Brasil nos leva a refletir sobre quais são os fatores responsáveis pelo fenômeno. As instituições de ensino agem para aprimorar as condutas das equipes médicas, reforçar as chamadas boas práticas clínicas e obter o aval de órgãos certificadores nacionais e internacionais²

Profissionalismo médico refere-se a atributos, valores, comportamentos, responsabilidadesecompromissos demédicos que são congruentes com as expectativas do público ${ }^{3}$. Desde a publicação da carta do profissionalismo em 2002, produto de vários anos de trabalho de líderes daAmerican Board of Internal Medicine (Abim), da American College of Physicians (ACP) e da Federação Europeia de Medicina Interna (European Federation of Internal Medicine), as quais estabeleceram os princípios fundamentais do profissionalismo médico, as discussões acerca da importância da inserção dele nos currículos e nas práticas se tornou cada vez mais frequente.Entre esses princípios, destacam-se: o princípio da primazia do bem-estar do paciente, compreendido como uma atuação que leve em consideração os interesses do paciente; o princípio da autonomia do paciente, o qual entende que os profissionais devem ser honestos com seus pacientes, empoderando-os para a tomada de decisões conscientes; e o princípio da justiça social, sendo a profissão médica responsável pela distribuição justa dos recursos de cuidado relacionado à saúde 4 . Dessa forma, o profissionallismo tornou-se uma nova competência que foi agregada ao conjunto de habilidades médicas, devendo ser demonstrada, ensinada e avaliada durante a formação desses profissionais ${ }^{5}$.

Éamplamente aceito que a avaliação formativa impulsiona o aprendizado e que incluir o profissionalismo no currículo motiva os alunos a se preparar para o atendimento ao paciente, sendo um elemento essencial na promoção da melhoria da qualidade desse atendimento ${ }^{6}$. A avaliação do profissionalismo é fundamental para a identificação e remediação de alunos cujo desempenho pode colocar em risco os pacientes ${ }^{7}$.

Em fevereiro de 1999, o Accreditation Council for Graduate Medical Education (ACGME), responsável pela acreditação de residências médicas nos Estados Unidos, definiu seis competências gerais comuns a todas as especialidades médicas e seus consequentes resultados, incluindo, entre elas, o profissionalismo ${ }^{8}$.

O Professionalism Mini-Evaluation Exercise (P-MEX) é um instrumento de avaliação do profissionalismo médico criado no Canadá, em 2006, na Universidade de McGill $^{9}$, e já foi validado no Japão, em $2011^{10}$ there is no standardized tool for its measurement. The authors sought to verify the validity, reliability, and generalizability of the Professionalism MiniEvaluation Exercise (P-MEX, na Finlândia, em 2015'11, e no Irã, em $2019^{12}$, mostrando evidências de validade, confiabilidade e reprodutibilidade dos resultados nesses países.

OBrasil ainda tem poucos estudos que abordam métodos de avaliação de profissionalismo médico ${ }^{13}$. Na literatura em língua portuguesa, não foi identificado nenhum instrumento que avalie o profissionalismo durante a formação médica. $\mathrm{O}$ contrato social que sustenta o profissionalismo médico pode ser enfraquecido quando importamos apenas documentos e instrumentos de avaliação de outros países. O que temos no Brasil referente ao tema é baseado, principalmente, no ensino e na avaliação do profissionalismo a partir de matrizes internacionais ${ }^{14}$ and has been recognized as a skill that should be developed by all health professionals. Objective: The aim of the present study was to assess professionalism among graduates of health-related courses on a worldwide scale. Methods: Systematic review following the recommendations of the Preferred Reporting Items for Systematic Reviews and Meta-Analyses (PRISMA.

Para possibilitar a padronização da avaliação do profissionalismo de médicos residentes no cenário brasileiro e o acompanhamento longitudinal ao longo da sua formação profissional, faz-se necessária a utilização de um instrumento adaptado para uso no país. O objetivo do presente trabalho é a tradução e a adaptação transcultural para a língua portuguesa do instrumento P-MEX, já utilizado em outros países para avaliação do profissionalismo.

\section{MÉTODOS}

Trata-se de uma pesquisa metodológica de tradução e adaptação transcultural do instrumento de avaliação de profissionalismo P-MEX que foi realizada no período de julho de 2019 a agosto de 2020. O P-MEX consta de 21 itens que contemplam quatro domínios, a saber: habilidades de relacionamento médico-paciente, habilidades reflexivas, gestão do tempo e habilidades de relacionamento interprofissional ${ }^{9}$ the Professionalism Mini-Evaluation Exercise (P-MEX. Embora uma versão mais longa de 24 itens tenha sido usada nos estudos de validação do P-MEX no Canadá e no Japão, os autores do instrumento apoiaram e orientaram o uso da versão de 21 itens.

A tradução e a adaptação transcultural do instrumento de avaliação de profissionalismo P-MEX, a fim de manter a equivalência conceitual, cultural, semântica e idiomática, foram realizadas de acordo com as diretrizes da International Test Commission (ITC) - segunda versão $2017^{15}$ (Figura 1). 
Figura 1. Fluxograma das etapas da tradução e adaptação transcultural do “Professionalism Mini-Evaluation Exercise (P-MEX)” para o português brasileiro

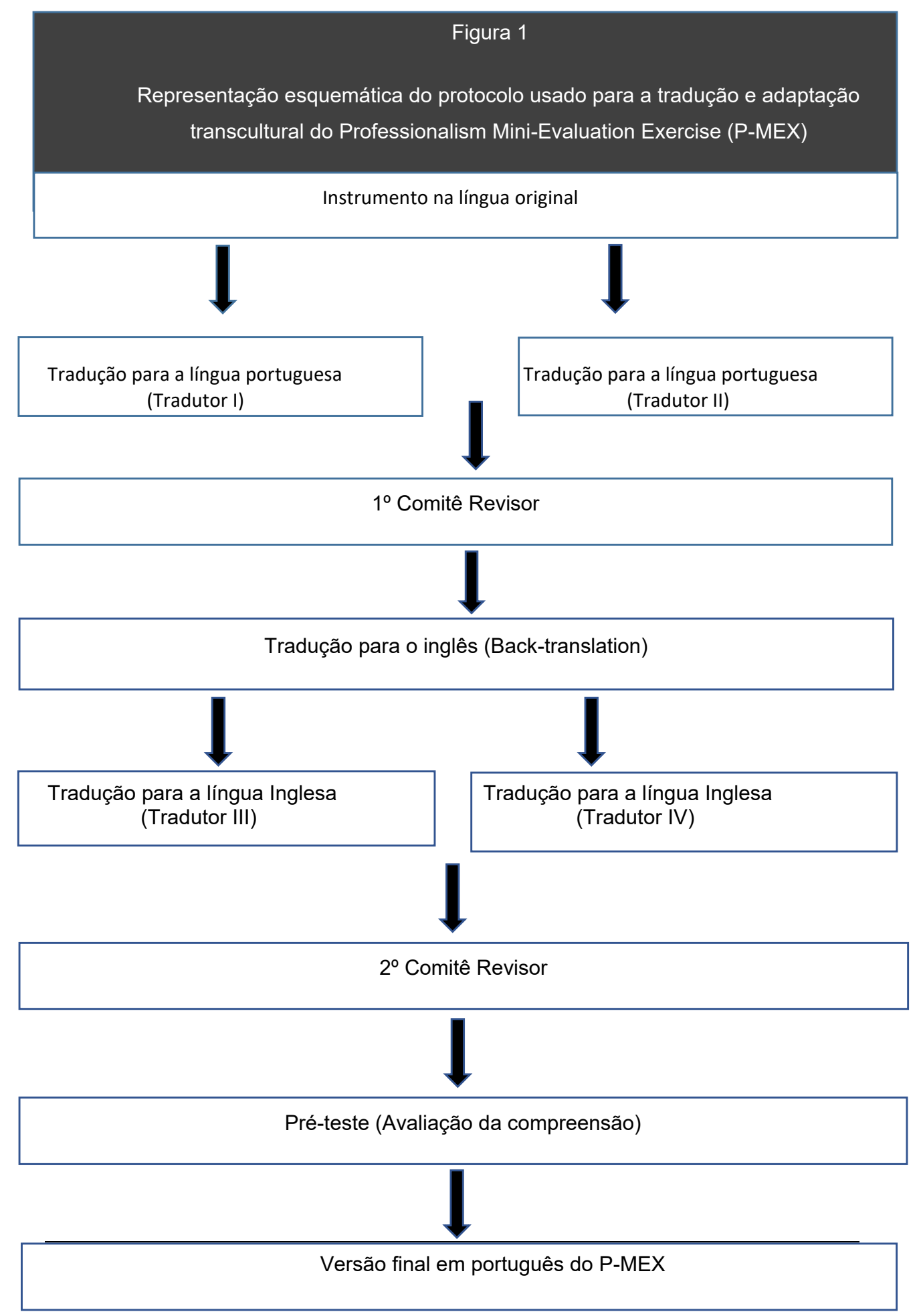

Fonte: Adaptado de Beaton et al $^{18}$.

A primeira etapa consistiu na solicitação da autorização do autor do P-MEX (Richard Cruess) para dar início ao processo de tradução e adaptação transcultural do instrumento. Após a autorização formal do autor, a etapa seguinte foi a tradução do instrumento original P-MEX para o português falado no Brasil. Essa tradução foi realizada de forma independente por dois médicos cuja língua materna é a língua portuguesa e que possuem fluência na língua inglesa (Quadro 1). Um comitê revisor, composto pelos dois médicos brasileiros que realizaram a tradução para o português com as pesquisadoras, reuniu-se e elaborou a primeira versão em português do P-MEX. 
Quadro 1.Tradução do questionário "Professionalism Mini-Evaluation Exercise (P-MEX)"

\begin{tabular}{|c|c|c|}
\hline Versão original & Tradução I & Tradução II \\
\hline Listened actively to patient & Escutado ativamente ao paciente & Escutou ativamente o paciente \\
\hline Showed interest in patient as a person & $\begin{array}{l}\text { Demonstrou interesse no paciente } \\
\text { como pessoa }\end{array}$ & $\begin{array}{l}\text { Mostrou interesse no paciente enquanto } \\
\text { pessoa }\end{array}$ \\
\hline Recognized and met patient needs & $\begin{array}{l}\text { Necessidades de paciente reconhecidas } \\
\text { e atendidas }\end{array}$ & $\begin{array}{l}\text { Reconheceu e atendeu às expectativas } \\
\text { do paciente }\end{array}$ \\
\hline Extended his/herself to meet patient needs & $\begin{array}{l}\text { Estendeu-se para atender às } \\
\text { necessidades dos pacientes }\end{array}$ & $\begin{array}{l}\text { Se esforçou para atender às expectativas } \\
\text { do paciente }\end{array}$ \\
\hline Ensured continuity of patient care & $\begin{array}{l}\text { Continuidade assegurada do cuidado } \\
\text { do paciente }\end{array}$ & $\begin{array}{l}\text { Assegurou a continuidade do cuidado } \\
\text { com o paciente }\end{array}$ \\
\hline Advocated on behalf of a patient & Defende em nome do paciente & Advogou em nome do paciente \\
\hline Demonstrated awareness of limitations & Demonstra consciência das limitações & $\begin{array}{l}\text { Demonstrou consciência das suas } \\
\text { limitações }\end{array}$ \\
\hline Maintained appropriate boundaries & Manteve limites apropriados & Manteve distanciamento apropriado \\
\hline $\begin{array}{l}\text { Maintained composure in a difficult } \\
\text { situation }\end{array}$ & $\begin{array}{l}\text { Compostura mantida em uma situação } \\
\text { difícil }\end{array}$ & $\begin{array}{l}\text { Manteve a compostura em uma situação } \\
\text { difícil }\end{array}$ \\
\hline Maintained appropriate appearance & Aparência apropriada mantida & Manteve a aparência apropriada \\
\hline Was on time & Estava na hora & Foi pontual \\
\hline Completed tasks in a reliable fashion & Tarefas concluídas de forma fiável & Completou as tarefas de forma confiável \\
\hline Addressed own gaps in knowledge and skills & $\begin{array}{l}\text { Abordou as próprias lacunas no } \\
\text { conhecimento e nas competências }\end{array}$ & $\begin{array}{l}\text { Abordou suas próprias lacunas em } \\
\text { conhecimentos e habilidades }\end{array}$ \\
\hline Was available to colleagues & Estava disponível para os colegas & Esteve disponível para os colegas \\
\hline
\end{tabular}

Essa versão em português do P-MEX, aprovado no primeiro comitê revisor, foi traduzida para a língua original do instrumento (back-translation). Essa etapa foi realizada por dois professores de inglês, nativos de países de língua inglesa, residentes no Brasil e fluentes na língua portuguesa. Os profissionais que realizaram a tradução retrógrada para a língua inglesa não tiveram acesso ao instrumento na sua versão original em inglês e foram orientados a fazer uma tradução literal da versão em português do P-MEX.

O segundocomitê revisor foi de suma importância para alcançar a equivalência transcultural e promoveu a elaboração de uma versão considerada consenso final. Esse comitê revisor foi constituído pelos dois professores de inglês (tradutores III e IV) e as pesquisadoras. Nessa etapa, o comitê revisor dispunha de todo material elaborado até o momento e verificou a ocorrência de equivalência semântica, idiomática e conceitual entre a versão original do instrumento e a versão consenso final. Vale lembar que essa versão consenso final ainda poderia passar por alterações após as avaliações práticas (pré-teste).

$\mathrm{O}$ instrumento traduzido para a língua portuguesa (apreciado pelo primeiro comitê revisor) e a retrotradução para a língua inglesa (apreciado pelo segundo comitê revisor) foram enviados para o autor por correspondência eletrônica, conforme sua solicitação inicial, para que ele pudesse fazer suas considerações. Ele aprovou a retrotradução na sua íntegra, sem necessidade de nenhuma modificação.

A etapa final consistiu na aplicação da versão consenso final do P-MEX. O objetivo dessa etapa foi avaliar a clareza, a compreensão e a aceitabilidade do instrumento traduzido em uma população semelhante à população-alvo, ou seja, médicos residentes em formação e preceptores de residência médica. Médicos residentes em pediatria em um hospital universitário de ensino preencheram um total de 12 formulários do P-MEX, aplicados por dois preceptores da residência de pediatria da 
mesma unidade. Buscou-se a todo momento identificar alguma parte do instrumento que pudesse gerar dúvidas e interpretações errôneas. Constatou-se que a versão consenso final proporcionou entendimento equivalente, compreensível e claro, consolidando-a como a versão final em português do P-MEX.

O presente estudo foi aprovado pelo Comitê de Ética em Pesquisa da instituição de ensino superior participante e está em conformidade com a Resolução $n^{\circ} 466 / 2012$. Todos os participantes formam orientados sobre o estudo e assinaram o Termo de Consentimento Livre e Esclarecido.

\section{RESULTADOS}

Em relação às traduções exibidas no Quadro 1, as discordâncias mais frequentes encontradas nas traduções foram relacionadas a palavras ou termos com significados semelhantes no Brasil (por exemplo: “Demonstrou interesse no paciente como pessoa" e "Mostrou interesse no paciente enquanto pessoa") e tempos verbais diferentes (por exemplo: "Demonstra consciência das limitações" e "Demonstrou consciência das suas limitações").

Durante a síntese das traduções, todas as discordâncias encontradas na etapa anterior foram estudadas, e os profissionais componentes do primeiro comitê revisor optaram pelo termo que consideraram mais usual no português falado no Brasil, para compor a versão consenso em português.

As versões retrotraduzidas para a língua inglesa foram muito parecidas, e as diferenças foram consideradas secundárias ao uso de palavras sinônimas. Dessa forma, o segundo comitê revisor optou por um retorno mais literal da tradução respeitando o discurso dentro dos padrões normais da cultura-alvo. Essa versão consenso da retrotradução foi enviada aos autores do instrumento que a aprovaram sem considerações adicionais e na íntegra.

A etapa final consistiu na aplicação da versão consenso final do instrumento a um público semelhante ao públicoalvo da pesquisa. Foram avaliados 12 residentes médicos de pediatria em um hospital de ensino, durante estágio de pronto-socorro e unidade de terapia intensiva (UTI) neonatal, por dois preceptores que já acompanhavam esses residentes. Após observação de um atendimento desses residentes por aproximadamente 15 minutos, os preceptores preencheram o instrumento e, em seguida, forneceram feedback cuja etapa teve como objetivo avaliar sua compreensão. Inferiuse que não houve impedimentos ao pleno entendimento do instrumento por todos os participantes, tanto residentes quanto preceptores. Buscou-se identificar alguma questão problemática, a fim de propiciar soluções para que o entendimento fosse mais claro e sem gerar dúvidas por parte das pessoas que fossem responder a ele. $O$ instrumento foi enviado aos preceptores para ser aplicado em residentes sob sua supervisão. Tais residentes foram orientados a fazer apontamentos de possíveis dúvidas ou sugestões de melhoras. Assim, discutiu-se em grupo (preceptores e pesquisadoras) item por item do questionário. Após esse processo, nenhuma dúvida foi levantada quanto à compreensão, considerando os itens corretamente compreendidos. Houve considerações apenas em relação à apresentação do instrumento, sendo sugerido que não fossem utilizadas abreviaturas na pontuação dos itens da escala (inaceitável, abaixo das expectativas, dentro das expectativas, excede expectativas e evento crítico).

Dessa forma, a partir da análise realizada, propôs-se a seguinte versão final em português do P-MEX (Quadro 2).

\section{DISCUSSÃO}

O instrumento "P-MEX" foi traduzido e adaptado de acordo com as diretrizes da ITC - segunda versão $2017^{15}$ para uso no Brasil, não sendo identificadas dificuldades de compreensão em nenhuma das questões. Todo o processo realizado, incluindo a aprovação final do autor do instrumento original, sugere que a nova versão adaptada tem validade de conteúdo correspondente à original.

Durante o processo de tradução e adaptação transcultural do instrumento, foram necessários pequenos ajustes gramaticais e culturais de modo a adaptá-lo ao contexto brasileiro ${ }^{16}$. As alterações mais frequentes encontradas no texto, introduzidas por meio da tradução, enquadraram-se nas três categorias mais encontradas na literatura: omissões, substituições ou acréscimos ${ }^{17}$. Durante 0 consenso das traduções, buscou-se a forma que caracterizasse melhor as versões discutidas e pensadas à luz dos objetivos do questionário original, de modo a atingir a equivalência semântica. Foram seguidas as recomendações práticas de tradução para a adaptação transcultural, principalmente no que se refere a usar voz ativa em vez da passiva, e evitar o modo subjuntivo e as formas possessivas ${ }^{18}$.

É sugerido que o formato do item, incluindo o layout físico, seja o mesmo nas duas versões do instrumento durante o processo ${ }^{19}$. Nessa tradução e adaptação transcultural, entretanto, optou-se por seguir a sugestão dos preceptores que aplicaram o pré-teste de retirar as siglas, e utilizar as palavras por extenso (não se aplicam "inaceitável", "abaixo das expectativas", "dentro das expectativas" e "excedeu expectativas"). Outra alteração feita foi a junção de itens por domínios. O P-MEX avalia quatro domínios, a saber: relação médico-paciente, relacionamento interpessoal, reflexão e manejo do tempo ${ }^{20}$. 
Quadro 2. Retrotradução do "Professionalism Mini-Evaluation Exercise (P-MEX)"

\begin{tabular}{|c|c|c|}
\hline Retrotradução 1 & Retrotradução 2 & Versão consenso (segundo comitê revisor) \\
\hline Actively listened to the patient & Actively listened to the patient & Escutou ativamente o paciente \\
\hline $\begin{array}{l}\text { Showed interest in the patient as a } \\
\text { person }\end{array}$ & Showed interest in the patient as a person & Mostrou interesse no paciente como pessoa \\
\hline Recognized and met patient needs & $\begin{array}{l}\text { Recognized and took care of the patient's } \\
\text { needs }\end{array}$ & $\begin{array}{l}\text { Reconheceu e atendeu às necessidades do } \\
\text { paciente }\end{array}$ \\
\hline Over and above to meet patient needs & Went beyond meeting the patient's needs & $\begin{array}{l}\text { Superou-se para atender às necessidades } \\
\text { do paciente }\end{array}$ \\
\hline Continued patient care was ensured & Constantly made sure of the patient's care & $\begin{array}{l}\text { Assegurou-se a continuidade do cuidado } \\
\text { com o paciente }\end{array}$ \\
\hline Advocated in defense of the patient & Advocated for the patient & Advogou em defesa do paciente \\
\hline $\begin{array}{l}\text { Demonstrated awareness of one's } \\
\text { limitations }\end{array}$ & Showed awareness of own limitations & $\begin{array}{l}\text { Demonstrou consciência das suas } \\
\text { limitações }\end{array}$ \\
\hline Maintained appropriate limits & Maintained appropriate boundaries & Manteve limites apropriados \\
\hline Kept composure in a difficult situation & $\begin{array}{l}\text { Remained composed in difficult } \\
\text { situations }\end{array}$ & $\begin{array}{l}\text { Manteve a compostura em uma situação } \\
\text { difícil }\end{array}$ \\
\hline Maintained proper appearance & Maintained appropriate appearance & Manteve aparência apropriada \\
\hline Was punctual & Was punctual & Foi pontual \\
\hline Has completed tasks reliably & Completed tasks reliably & Completou as tarefas de maneira confiável \\
\hline $\begin{array}{l}\text { Addressed one's own knowledge and } \\
\text { skills gaps }\end{array}$ & $\begin{array}{l}\text { Addressed own gaps in knowledge and } \\
\text { skills }\end{array}$ & $\begin{array}{l}\text { Abordou suas próprias lacunas de } \\
\text { conhecimento e habilidades }\end{array}$ \\
\hline Was available to colleagues & Was available for colleagues & Estava disponível para os colegas \\
\hline
\end{tabular}

A aplicação do pré-teste permitiu que os preceptores respondessem ao instrumento e o avaliassem posteriormente quanto ao entendimento, à aparência, à clareza e à escrita. Também foram incentivados a fornecer sugestões de melhorias quando considerassem pertinentes. Isso garantiu que a versão adaptada mantivesse sua equivalência na aplicada.

A equivalência semântica, que é a adequada transferência de significados entre idiomas, alcançando efeito similar durante sua aplicação em diferentes línguas, foi comprovada durante a tradução e adaptação transcultural do instrumento original. Um aspecto relevante da equivalência semântica é que o nível da linguagem usada demonstrou ser apropriado à populaçãoalvo. Um aspecto não menos importante referiu-se ao fato de, durante o pré-teste (avaliação da compreensão), haver ainda, equivalência operacional. O tempo médio para responder ao questionário foi de 15 minutos, dentro da média gasta no instrumento original, que é de 15 a 20 minutos 9 .
A aplicação desse instrumento fornecerá contribuições na avaliação sobre o nível de profissionalismo do médico residente, permitindo analisar adequadamente seu desempenho profissional durante toda a sua formação e, quiçá, possibilitando interferências nas discrepâncias observadas pelos preceptores ${ }^{21,22}$ mandated by accrediting bodies. Responding to a call for comprehensive research on remediation of student professionalism lapses, the authors explored current medical school policies and practices. Method In 2012-2013, key administrators at U.S. and Canadian medical schools accredited by the Liaison Committee on Medical Education were interviewed via telephone or e-mail. The structured interview questionnaire contained open-ended and closed questions about practices for monitoring student professionalism, strategies for remediating lapses, and strengths and limitations of current systems. The authors employed a mixed-methods approach, using descriptive statistics and qualitative analysis based on grounded theory. 
Results Ninety-three (60.8\%. Dessa forma, o instrumento poderá ser utilizado como objeto de melhoria do profissionalismo ${ }^{23}$. Além disso, trata-se de uma ferramenta de avaliação direta, rápida e de fácil execução, cuja aplicação, pode ser estendida ao estudante de Medicina.

Já está em andamento o processo de validação estatística quantitativa do P-MEX, pois, como se trata da tradução de instrumento de mensuração, ele deve manter a capacidade de mensurar conforme o original.

\section{CONCLUSÃO}

Diante da lacuna de instrumentos para medir o profissionalismo de médicos residentes no Brasil em suas rotinas de atendimentos, realizaram-se a tradução e a adaptação transcultural do P-MEX para uso no país. O seguimento de etapas de acordo com o referencial metodológico escolhido foi importante para garantir a qualidade no resultado final. Esse instrumento poderá ser utilizado tanto para avaliação do comportamento profissional de residentes médicos como para o planejamento de suas atividades de educação. Na perspectiva do ensino, o P-MEX poderá ser útil com a finalidade de instigar os estudantes a buscar agir de acordo com o esperado para uma prática profissional mais adequada e com menos risco para os pacientes e a sociedade.

\section{AGRADECIMENTOS}

Agradecemos às preceptoras Paula Arataque Roriz Steckelberg e Fernanda Dornela de Melo, que aplicaram o instrumento durante a realização do pré-teste, e à professora doutora Maria Goretti Queiroz, que muito nos incentivou na realização deste artigo.

\section{CONTRIBUIÇÃO DAS AUTORAS}

Mariana Matias de Lima Holdefer e Cláudia Fonseca Sena participaram, de forma igualitária, do planejamento do estudo, da revisão bibliográfica, da coleta e análise de dados, da composição dos comitês de revisão, e da redação de todas as versões do artigo. Alessandra Vitorino Naghettini e Edna Regina Silva Pereira participaram do planejamento do artigo, da revisão bibliográfica e da correção da redação do artigo.

\section{CONFLITO DE INTERESSES}

As autoras declaram não haver conflito de interesses neste estudo.

\section{FINANCIAMENTO}

Declaramos que não houve financiamento para a realização desta pesquisa.

\section{REFERÊNCIAS}

1. Conselho Nacional de Justiça. Judicialização da saúde no Brasil: perfil das demandas, causas e propostas de solução. Brasília: Insper; 2019.

2. David BP, Ana CSC, Lucas ELFSM, Tadeu PL, Lorenna PLCMC. Erro médico e prevenção de ações judiciais: análise dos deveres anexos na relação médico-paciente para além da assistência técnica. Braz J Health Rev. 2020;3(4):8717-31.

3. Paltiel O, Lowenstein L, Demma J, Manor O. International workshop on "professionalism in the practice of medicine - where are we now?" Isr J Health Policy Res. 2017;6(1):1-7.

4. American Board of Internal Medicine. Project Professionalism Philadelphia: Abim; 1995.

5. Miles PV, Conway PH, Pawlson LG. Physician professionalism and accountability: the role of collaborative improvement networks. Pediatrics. 2013;131(Suppl 4):S204-9.

6. Carneiro MA, Cunha SM, Feitosa ES, Sá RB, Brilhante AVM. O profissionalismo e suas formas de avaliação em estudantes de Medicina: uma revisão integrativa. Interface Comun Saúde Educ. 2020;24:e190126.

7. Deitte L. The new residency curriculum: professionalism, patient safety, and more. J Am Coll Radiol. 2013;10(8):613-7. doi: 10.1016/j.jacr.2013.01.027.

8. Accreditation Council for Graduate Medical Education - International Requir Process Overv. 2015;11(1):2-4 [acesso em 29 jan 2019]. Disponível em: http://www.acgme-i.org.

9. Cruess R, Mcllroy JH, Cruess S, Ginsburg S, Steinert Y. The Professionalism Mini-evaluation Exercise: a preliminary investigation. Acad Med 2006;81(10 Suppl):S74-8 [acesso em 29 jan 2019]. Disponível em: http:// www.ncbi.nlm.nih.gov/pubmed/17001141.

10. Tsugawa $\mathrm{Y}$, Ohbu S, Cruess $\mathrm{R}$, Cruess $\mathrm{S}$, Okubo $\mathrm{T}$, Takahashi $\mathrm{O}$, et al. Introducing the professionalism mini-evaluation exercise ( $p-M E X)$ in Japan: results from a multicenter, cross-sectional study. Acad Med. 2011;86(8):1026-31.

11. Karukivi M, Kortekangas-Savolainen O, Saxén U, Haapasalo-Pesu K-M Professionalism Mini-Evaluation Exercise in Finland: a preliminary investigation introducing the Finnish version of the P-MEX instrument. Adv Med Educ Prof. 2015;3(4):154-8 [acesso em 29 jan 2019]. Disponível em: http://www.ncbi.nlm.nih.gov/pubmed/26457311\%0Ahttp://www. pubmedcentral.nih.gov/articlerender.fcgi?artid=PMC4596380.

12. Amirhajlou L, Bidari A, Alipour F, Yaseri M, Vaziri S, Rezaie, M et al. Validity, reliability and acceptability of Professionalism Mini-Evaluation Exercise (P-MEX) for emergency medicine residency training. J Med Ethics Hist Med. 2019;12(12) [acesso em 29 jan 2019]. Disponível em: https://jmehm. tums.ac.ir/index.php/jmehm/article/view/508.

13. Carneiro MA, Cunha SDM, Feitosa ES, Veras A, Brilhante M. O profissionalismo e suas formas de avaliação em estudantes de Medicina: uma revisão integrativa. 2020:1-15. [acesso em 29 jan 2019].

14. Mendonça ET, Cotta RMM, Lelis VP, Carvalho Junior PM. Assessment of professionalism in students of health-related courses: a systematic review. Interface Commun Heal Educ. 2016;20(58):679-90.

15. International Test Commission. Guidelines for translating and adapting tests. 2nd ed. ITC; 2017

16. Arthur JP, Mantovani MF, Ferraz MIR, Mattei ÂT, Kalinke LP, Corpolato RC Translation and cross-cultural adaptation of the hypertension knowledgelevel scale for use in Brazil. Rev Lat Am Enfermagem. 2018;26:e3073.

17. Fortes CPDD, Araújo APQC. Check list para tradução e adaptação transcultural de questionários em saúde. Cad Saude Colet. 2019;27(2):202-9.

18. Beaton DE, Bombardier C, Guillemin F, Ferraz MB. Guidelines for the process of cross-cultural adaptation of self-report measures. Spine (Phila Pa 1976). 2000;25(24):3186-91 [acesso em 29 jan 2019]. Disponível em: http://www.ncbi.nlm.nih.gov/pubmed/11124735.

19. Hambleton RK, Zenisky AL. Translating and adapting tests for cross cultural assessments. Cross-Cultural Res Methods Psychol. 2010;46-70.

20. Fong W, Kwan YH, Yoon S, Phang JK, Thumboo J, Leung YY, et al. Assessment of medical professionalism: preliminary results of a qualitative study. BMC Med Educ. 2020;20(27) 
21. Ziring D, Danoff D, Grosseman S, Langer D, Esposito A, Jan MK, et al. How do medical schools identify and remediate professionalism lapses in medical students? A study of U. S. and Canadian Medical Schools. Acad Med. 2015;90(7):913-20.
22. Mak-vanVM,Teherani A, van MookW,CroisetG, KusurkarRA. How to identify, address and report students' unprofessional behaviour in medical school. Med Teach. 2020;42(4):372-9. doi: 10.1080/0142159X.2019.1692130.

23. Maricoto T. A necessidade do ensino do profissionalismo. Rev Port Clínica Geral. 2019;35(4):258-60.

\section{ERRATA}

Na página 2, onde se lê:

[...] foi validado no Japão, em $2011^{10}$ there is no standardized tool for its measurement. The authors sought to verify the validity, reliability, and generalizability of the Professionalism Mini-Evaluation Exercise (P-MEX, na Finlândia, em 2015 11, e no Irã, em 201912, mostrando evidências de validade, confiabilidade e reprodutibilidade dos resultados nesses países.

O Brasil ainda tem poucos estudos que abordam métodos de avaliação de profissionalismo médico ${ }^{13}$. Na literatura em língua portuguesa, não foi identificado nenhum instrumento que avalie o profissionalismo durante a formação médica. O contrato social que sustenta o profissionalismo médico pode ser enfraquecido quando importamos apenas documentos e instrumentos de avaliação de outros países. O que temos no Brasil referente ao tema é baseado, principalmente, no ensino e na avaliação do profissionalismo a partir de matrizes internacionais ${ }^{14}$ and has been recognized as a skill that should be developed by all health professionals. Objective: The aim of the present study was to assess professionalism among graduates of health-related courses on a worldwide scale. Methods: Systematic review following the recommendations of the Preferred Reporting Items for Systematic Reviews and Meta-Analyses (PRISMA.

Para possibilitar a padronização da avaliação do profissionalismo de médicos residentes no cenário brasileiro e o acompanhamento longitudinal ao longo da sua formação profissional, faz-se necessária a utilização de um instrumento adaptado para uso no país. O objetivo do presente trabalho é a tradução e a adaptação transcultural para a língua portuguesa do instrumento P-MEX, já utilizado em outros países para avaliação do profissionalismo.

\section{MÉTODOS}

Trata-se de uma pesquisa metodológica de tradução e adaptação transcultural do instrumento de avaliação de profissionalismo P-MEX que foi realizada no período de julho de 2019 a agosto de 2020. O P-MEX consta de 21 itens que contemplam quatro domínios, a saber: habilidades de relacionamento médico-paciente, habilidades reflexivas, gestão do tempo e habilidades de relacionamento interprofissional9the Professionalism Mini-Evaluation Exercise (P-MEX. Embora uma versão mais longa de 24 itens tenha sido usada nos estudos de validação do P-MEX no Canadá e no Japão, os autores do instrumento apoiaram e orientaram o uso da versão de 21 itens.

\section{Considera-se:}

[...] foi validado no Japão, em $2011^{10}$, na Finlândia, em 2015 ${ }^{11}$, e no Irã, em 201912, mostrando evidências de validade, confiabilidade e reprodutibilidade dos resultados nesses países.

O Brasil ainda tem poucos estudos que abordam métodos de avaliação de profissionalismo médico ${ }^{13}$. Na literatura em língua portuguesa, não foi identificado nenhum instrumento que avalie o profissionalismo durante a formação médica. O contrato social que sustenta o profissionalismo médico pode ser enfraquecido quando importamos apenas documentos e instrumentos de avaliação de outros países. $O$ que temos no Brasil referente ao tema é baseado, principalmente, no ensino e na avaliação do profissionalismo a partir de matrizes internacionais ${ }^{14}$.

Para possibilitar a padronização da avaliação do profissionalismo de médicos residentes no cenário brasileiro e o acompanhamento longitudinal ao longo da sua formação profissional, faz-se necessária a utilização de um instrumento adaptado para uso no país. O objetivo do presente trabalho é a tradução e a adaptação transcultural para a língua portuguesa do instrumento P-MEX, já utilizado em outros países para avaliação do profissionalismo.

\section{MÉTODOS}

Trata-se de uma pesquisa metodológica de tradução e adaptação transcultural do instrumento de avaliação de 
profissionalismo P-MEX que foi realizada no período de julho de 2019 a agosto de 2020. O P-MEX consta de 21 itens que contemplam quatro domínios, a saber: habilidades de relacionamento médico-paciente, habilidades reflexivas, gestão do tempo e habilidades de relacionamento interprofissional ${ }^{9}$. Embora uma versão mais longa de 24 itens tenha sido usada nos estudos de validação do P-MEX no Canadá e no Japão, os autores do instrumento apoiaram e orientaram o uso da versão de 21 itens.

\section{Na página 6, onde se lê:}

A aplicação desse instrumento fornecerá contribuições na avaliação sobre o nível de profissionalismo do médico residente, permitindo analisar adequadamente seu desempenho profissional durante toda a sua formação e, quiçá, possibilitando interferências nas discrepâncias observadas pelos preceptores ${ }^{21,22}$ mandated by accrediting bodies. Responding to a call for comprehensive research on remediation of student professionalism lapses, the authors explored current medical school policies and practices. Method In 2012-2013, key administrators at U.S. and Canadian medical schools accredited by the Liaison Committee on Medical Education were interviewed via telephone or e-mail. The structured interview questionnaire contained open-ended and closed questions about practices for monitoring student professionalism, strategies for remediating lapses, and strengths and limitations of current systems. The authors employed a mixed-methods approach, using descriptive statistics and qualitative analysis based on grounded theory.

\section{Considera-se:}

A aplicação desse instrumento fornecerá contribuições na avaliação sobre o nível de profissionalismo do médico residente, permitindo analisar adequadamente seu desempenho profissional durante toda a sua formação e, quiçá, possibilitando interferências nas discrepâncias observadas pelos preceptores ${ }^{21,22}$.

Na página 7, onde se lê:

Results Ninety-three $(60.8 \%$. Dessa forma, o instrumento poderá ser utilizado como objeto de melhoria do profissionalismo ${ }^{23}$. Além disso, trata-se de uma ferramenta de avaliação direta, rápida e de fácil execução, cuja aplicação, pode ser estendida ao estudante de Medicina.

\section{Considera-se:}

Dessa forma, o instrumento poderá ser utilizado como objeto de melhoria do profissionalismo ${ }^{23}$. Além disso, trata-se de uma ferramenta de avaliação direta, rápida e de fácil execução, cuja aplicação, pode ser estendida ao estudante de Medicina.

Revista Brasileira de Educação Médica, 46(1): e062, 2022. 